\title{
Pessary Dosage Form Category
}

National Cancer Institute

\section{Source}

National Cancer Institute. Pessary Dosage Form Category. NCI Thesaurus. Code C154591.

A type of solid pharmaceutical dose form consisting of a material that is usually ovoid and formed by moulding, of a suitable volume and consistency for insertion into the vagina where it dissolves, disperses or melts. 